\title{
Newborn bloodspot screening for Duchenne Muscular Dystrophy: 21 years experience in Wales (UK)
}

\begin{abstract}
Stuart J Moat ${ }^{\star, 1}$, Donald M Bradley ${ }^{1}$, Rachel Salmon ${ }^{1}$, Angus Clarke ${ }^{2}$ and Louise Hartley ${ }^{3}$
Duchenne muscular dystrophy (DMD), a progressive X-linked neuromuscular disorder, has an estimated worldwide incidence of 1:3500 male births. Currently, there are no curative treatments and the mean age of diagnosis is 5 years. In addition, subsequent pregnancies frequently occur before a diagnosis is made in an index case. An 'opt in' screening programme was introduced in Wales in 1990 with the aim to: reduce the diagnostic delay, permit reproductive choice and allow planning of the care of the affected boy. Newborn bloodspots were collected routinely as part of the Wales newborn screening programme. Specific consent was obtained for this test separately from the other tests. During the 21-year period, 369780 bloodspot cards were received from male infants, of these $343170(92.8 \%)$ were screened using a bloodspot creatine kinase (CK) assay following parental consent. A total of 145 cases had a raised CK activity ( $\geq 250 \mathrm{U} / \mathrm{l})$ and at follow-up, at 6-8 weeks of age, 79 cases had a normal serum CK (false-positive rate $0.023 \%$ ) and 66 cases had an elevated serum CK. DMD was confirmed in 56 cases by genotyping/muscle biopsy studies, Becker muscular dystrophy in 5 cases and other rarer forms of muscular dystrophy in 5 cases. This long-term study has so far identified 13 false-negative cases. The incidence of DMD in Wales of 1:5136 during this period is lower than that of 1:4046 before commencement of screening in Wales. Screening has reduced the diagnostic delay enabling reproductive choice for parents of affected boys and earlier administration of current therapies.
\end{abstract}

European Journal of Human Genetics (2013) 21, 1049-1053; doi:10.1038/ejhg.2012.301; published online 23 January 2013

Keywords: Duchenne muscular dystrophy; newborn screening; bloodspots; creatine kinase; false negatives

\section{INTRODUCTION}

Duchenne muscular dystrophy (DMD) is a progressive, lethal $\mathrm{X}$-linked neuromuscular disorder principally affecting males. The estimated worldwide incidence of DMD is approximately 1:3500 male live births, and affected boys have markedly elevated creatine kinase (CK) activities. ${ }^{1}$ The mean age for diagnosis has remained unchanged over many years at $4.5-5.5$ years. $^{2-5}$ It is frequently reported that parents had sought medical advice before this (mean delay from presentation to diagnosis is about 2 years), ${ }^{3}$ resulting in diagnostic delay and causing considerable anxiety and distress. ${ }^{6,7}$

The development of blood spot CK assays gave the potential for newborn boys to be screened for DMD. ${ }^{7,8}$ However, there were misgivings about the potential for harm being caused by newborn screening as there was no curative treatment. This prompted debate in medical journals and efforts to find alternative ways to bring forward the time of diagnosis in other ways. ${ }^{9}$ However, the evidence of family harm from late diagnoses, ${ }^{6,10}$ and the persistence of diagnostic delays ${ }^{4,5}$ has led to several different screening programmes being piloted across the world during the last 4 decades, using blood spot CK testing. ${ }^{11}$ Different screening protocols have been used to fit with the constraints of the health-care infrastructure within each of the individual countries; New Zealand, ${ }^{12}$ Scotland, ${ }^{13}$ France, ${ }^{14}$ Germany, ${ }^{15}$ Canada, ${ }^{16}$ Brazil/USA, ${ }^{17}$ Austria, ${ }^{18}$ Cyprus,${ }^{19}$ Belgium ${ }^{20}$ and USA (Ohio). ${ }^{21}$

Widespread bloodspot screening has not been adopted and currently no country nationally screens for DMD at birth. DMD does not fulfil traditional screening criteria as there is currently no curative treatment for DMD. ${ }^{22}$ However, the importance of an early diagnosis has increased, as the benefits of steroid treatment in delaying the loss of ambulation and improving quality of life has become clearer, ${ }^{23-25}$ so that a delayed diagnosis (occasionally as late as 8 years) may now be seen as preventing access to optimal medical management as well as optimal physiotherapy. ${ }^{26}$ In addition, social change has led to greater emphasis being given to the broader benefits to the individual and the family of an early diagnosis for any serious disorder, so that the purely 'medical' benefits are now understood as only one of a range of consequences resulting from a diagnosis. ${ }^{27}$

Here we report the experience of newborn bloodspot screening for DMD in Wales (United Kingdom) over a 21-year period. The initial aims of the newborn screening programme were: to avoid the anxiety associated with diagnostic delay; to give families reproductive choice in future pregnancies; and to enable families to plan for a future with a child with a disability. However, recent evidence suggests that an earlier diagnosis would allow timely intervention with existing therapies leading to long-term benefits. ${ }^{25}$ In addition, new approaches to therapy using exon-skipping drugs have recently been reported which have shown the potential to be disease modifying. ${ }^{28}$ With these, and other rational therapies on the horizon, there is renewed interest in the potential of population newborn bloodspot screening for DMD.

The Wales newborn screening programme for DMD was terminated in December 2011, as the UK Clinical Pathology Accreditation Service was unable to support the accreditation of the DMD screening testing service following the withdrawal of the external quality

${ }^{1}$ Wales Newborn Screening Laboratory, Department of Medical Biochemistry and Immunology, University Hospital Wales, Cardiff, Wales, UK; ${ }^{2}$ Department of Medical Genetics, University Hospital Wales, Cardiff, Wales, UK; ${ }^{3}$ Department of Paediatrics, University Hospital Wales, Cardiff, Wales, UK

${ }^{*}$ Correspondence: Dr SJ Moat, Wales Newborn Screening Laboratory, Department of Medical Biochemistry and Immunology, University Hospital Wales, Heath Park, Cardiff, Wales CF14 4XW, UK. Tel: + 44 (0)29 20743 562; Fax: + 44 (0)29 20744 065; E-mail: Stuart.Moat@Wales.nhs.uk

Received 29 October 2012; revised 12 December 2012; accepted 18 December 2012; published online 23 January 2013 
assurance programme by the Centres for Disease Control and Prevention in the United States. The external quality assurance programme was withdrawn because of a lack of sufficient participants to support a viable scheme. The cessation of the Wales newborn screening programme for DMD has prompted us to review the experience of bloodspot screening for DMD in Wales to help inform future policy on newborn bloodspot screening for DMD and for the screening of disorders to reduce the 'diagnostic odyssey'.

\section{MATERIALS AND METHODS}

\section{Bloodspot CK analysis}

Bloodspot CK determination was performed using an enzyme coupled end point reaction with the formation of nicotinamide adenine dinucleotide phosphate detected fluorimetrically as outlined by Orfanos and Naylor. ${ }^{8}$ During the period of 1990 to 1997 , a semi-quantitative test was used. ${ }^{29}$ From 1998 to December 2011, a quantitative test was performed on all bloodspot samples. This method was based on the same assay procedure and used 96-well microtitre plates using fluorescence plate readers (from 1998 to 2009 using a Wallac system - PerkinElmer Inc. (Waltham, MA, USA) and from 2009 to 2011 using a Twinkle LB 970 - Berthold Technologies, Herts, UK). All chemicals used in the assay were obtained from Sigma-Aldrich, Dorset, UK. A standard source of CK was obtained from Roche Diagnostics, Welwyn Garden City, UK (rabbit muscle CK). The Wales Newborn Screening Laboratory utilised QC material obtained from CDC (Atlanta, GA, USA) and participated in the CDC External Quality Assurance Programme for Bloodspot CK.

A screen-positive referral cutoff of $\geq 250 \mathrm{U} / 1$ whole blood was used and was derived from the analysis of anonymised bloodspots. ${ }^{29}$ During the evaluation period of the Twinkle Fluorimeter in 2009, anonymised bloodspots from 3346 male infants were analysed to confirm the current cutoff of $\geq 250 \mathrm{U} / \mathrm{l}$. The 99.9th centile was calculated to be $259 \mathrm{U} / \mathrm{l}$. The between assay CV at four different levels of CK activity was $<13 \%$. An analytical cutoff was set at $\geq 200 \mathrm{U} / \mathrm{l}$ to allow for variation in the assay and to allow for the variability in blood volume applied to the bloodspot card.

\section{Screening protocol}

The Wales Newborn Bloodspot DMD Screening protocol is shown in Figure 1. This protocol was designed to be compatible with the pattern of health-care delivery in the United Kingdom, with frequent home visits by a community midwife following a birth at home or discharge from hospital. Bloodspots were collected between days 5 and 8 of life as part of the Wales newborn bloodspot screening programme. Written consent for testing of DMD was obtained separately from the other newborn screening tests. The documentation of consent has varied, but for the last 13 years of screening this was standardised as signed parental consent on the newborn screening card. Those infants with test results that were not indicative of DMD or for any of the other disorders routinely screened for were then reported as part of the routine reporting of newborn screening results to Child Health Departments across Wales. Parents were informed of the results when the infant was 6-8 weeks of age by the health visitor

Those blood spot samples with an increased CK activity $(\geq 200 \mathrm{U} / \mathrm{l})$ underwent repeat analysis in duplicate. If the mean of the triplicate results was $\geq 250 \mathrm{U} / 1$ then a clinical referral and follow-up appointment was arranged. The details of the protocol were developed reflexively over the early months of the programme; ${ }^{29}$ the deferral of family notification and of venous blood sampling until around 6-8 weeks after the birth was introduced to allow time for the family to settle with the new child before the diagnosis of DMD was raised as a possibility. The newborn screening coordinator would then contact the family general practitioner and health visitor and the local paediatrician to arrange an appointment to meet with the family. The family were then contacted the day before the appointment to minimise the interval between notifying the parents of the possibility of the child having DMD and the final result of the venous CK measurement. On the day of the appointment, the paediatrician and health visitor explained the results from the newborn bloodspot screening test and a venous blood sample was then collected for

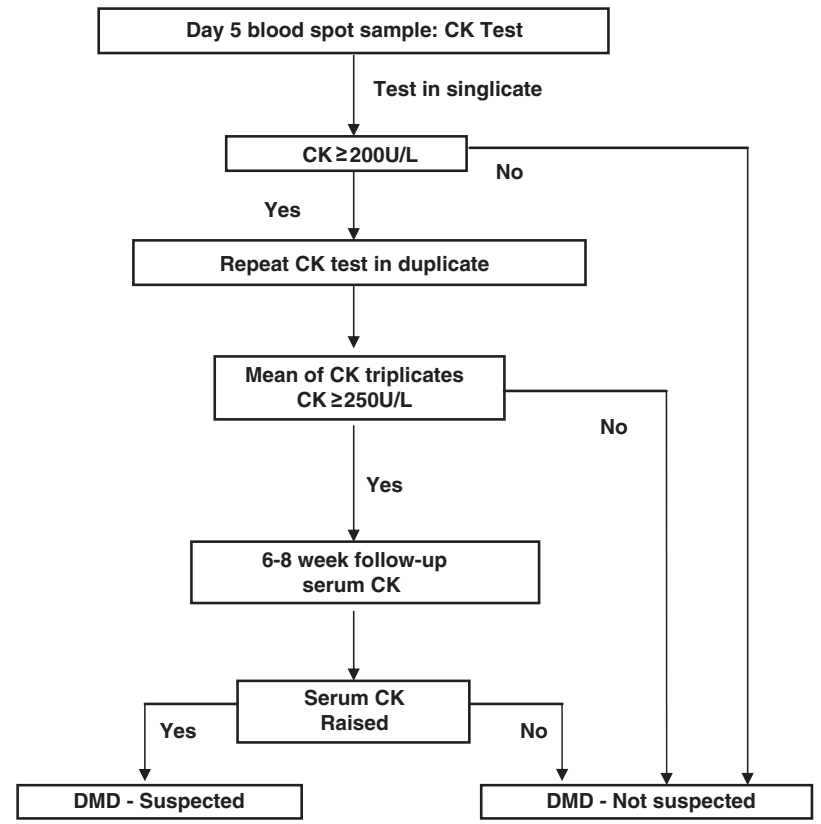

Figure 1 Wales DMD newborn bloodspot screening algorithm.

serum CK analysis. A sample would then often also be collected for possible future molecular genetic investigations.

Analysis of the serum/plasma sample for CK was pre-arranged with the local clinical laboratory and results were made available on the same day as the consultation to reduce parental anxiety. If the serum/plasma CK result was normal the parents were then informed and given the opportunity for further discussion. If the serum CK was elevated, further investigations (ie, genotyping and muscle biopsy) were then discussed with the parents.

An evaluation of the protocol for the consent process, handling of screenpositive results and other psychosocial implications of this programme have been reported previously. ${ }^{30-32}$

\section{RESULTS}

Figure 2 shows the results of the screening programme during the 21-year period (1990-2011). A total of 369780 newborn screening blood spot cards were received into the laboratory from boys born in Wales. Of these, 343170 (92.8\%) were tested for bloodspot CK following parental consent, $21942(5.9 \%)$ cards were marked as declined by the parents and $4668(1.3 \%)$ cards were defaulted in that the parents had neither consented nor declined to have their son screened. The proportion of infant boys whose parents declined the test was relatively constant over the 21 years at $\sim 5-6 \%$.

Of those bloodspot samples analysed, 145 had an increased CK activity $(0.042 \%)$. At the $6-8$ week follow-up, 79 of these cases were deemed as having a transient raised $\mathrm{CK}$, based on a normal serum CK activity giving a false-positive rate (FPR) of $0.023 \%$. One case was a screened female (the midwife had gained consent and indicated that the baby was a male), the bloodspot CK was $2247 \mathrm{U} / \mathrm{l}$ and at follow-up at 6 weeks of life the serum CK was normal at $108 \mathrm{U} / \mathrm{l}$. In all, 66 boys had an increased CK at the follow-up appointment, 56 were confirmed to have DMD, 5 had Becker muscular dystrophy and 5 were confirmed to have other rarer forms of muscular dystrophy including 2 cases of merosin-negative muscular dystrophy, 1 case of sarcoglycanopathy and 2 cases of undiagnosed muscular dystrophy.

Serum CK concentrations of the screen-positive cases at the 6-8 week follow-up are shown in Table 1. The mean serum CK in those boys confirmed to have Becker muscular dystrophy or other rarer 


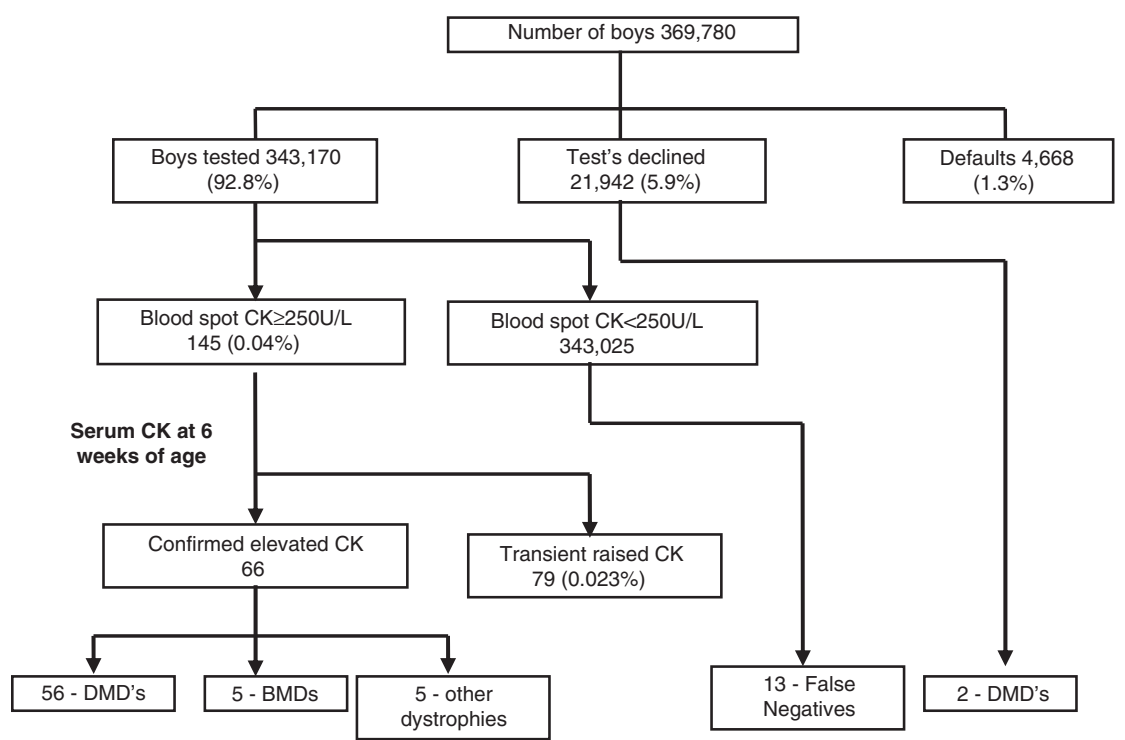

Figure 2 Overview of DMD screening results of the Wales newborn bloodspot screening programme for DMD (1990-2011).

Table 1 Serum CK activities in all of the screen-positive cases at the 6- to 8-week follow-up appointment

\begin{tabular}{lcccc}
\hline & \multicolumn{4}{c}{ Serum CK activities (U/I) } \\
\cline { 2 - 5 } & Mean & Median & Lowest & Highest \\
\hline DMD $(n=56)$ & 8052 & 8200 & 2442 & 14200 \\
BMD $(n=5)$ & 5595 & 3933 & 780 & 13732 \\
Other dystrophies $(n=5)$ & 4205 & 2162 & 735 & 13000 \\
Transient cases $(n=79)$ & 121 & 115 & 31 & 280 \\
\hline
\end{tabular}

forms of muscular dystrophy were lower than those observed in the DMD group. During the period of December 1998 to 2011, quantitative bloodspot CK results ( $n=93$ screen-positive cases) were obtained. Those DMD cases genetically confirmed had a mean bloodspot CK of $1123 \mathrm{U} / \mathrm{l}(n=36$, range 371-2406 U/l). Those cases confirmed to have had a transient elevation of $\mathrm{CK}$, based on a normal follow-up serum CK activity, had a mean bloodspot CK of $481 \mathrm{U} / 1$ (SD 354, $n=57$, range 251-2021 U/1). No correlation was observed between the initial screening bloodspot CK and the serum CK activity at the follow-up appointment.

To date, 13 false-negative cases have been identified (Table 2). In these false-negative cases, the mean and median age at time of initial serum/plasma CK was 3.95 and 2.93 years, respectively, (range 1.4-7.85 years) and the mean and median age at definitive diagnosis was 4.33 and 3.61 years, respectively, (range 1.9-8.6 years). The mean serum CK activity of this group at follow-up was $18673 \mathrm{U} / 1$ (range 6000-37 296 U/1).

In addition, two cases of DMD were diagnosed where the parents had declined screening; in the first case there was a family history of DMD and the serum CK at day 9 of life was $1740 \mathrm{U} / \mathrm{l}$, the second case was diagnosed at 4.5 years with motor difficulties and with a serum CK of 14000 U/l. Furthermore, an affected boy diagnosed at the age of 7.5 years was found not to have been screened for DMD as the card had been marked as female and was therefore not analysed for CK.

Overall, the screening programme had a sensitivity of $81.6 \%$, specificity of $99.97 \%$ and a positive predictive value (PPV) of $38.6 \%$.
Table 2 Details of the false-negative cases of DMD identified during the period July 1990-November 2011

\begin{tabular}{|c|c|c|c|c|c|c|}
\hline Case & $\begin{array}{l}\text { Clinical } \\
\text { presentation }\end{array}$ & $\begin{array}{c}\text { Age at } \\
\text { initial CK } \\
\text { test (years) }\end{array}$ & $\begin{array}{l}\text { Initial } \\
\text { serum } \\
\text { CK U/I }\end{array}$ & $\begin{array}{c}\text { Age at defini- } \\
\text { tive diagnosis } \\
\text { (years) }\end{array}$ & Genotype & $\begin{array}{l}\text { Age at } \\
\text { LOA } \\
\text { (years) }\end{array}$ \\
\hline 1 & Global delay & 3.0 & 32004 & 3.3 & $1-44$ & 8.3 \\
\hline 2 & Motor delay & 1.4 & 28000 & 1.9 & 54-59 del & 7.5 \\
\hline 3 & Global delay & 2.8 & 37296 & 2.9 & $\begin{array}{l}\text { Nonsense } \\
\text { exon } 64\end{array}$ & 10.0 \\
\hline 4 & Motor delay & 3.7 & 12300 & 3.9 & $51 \mathrm{del}$ & 9.3 \\
\hline 5 & $\begin{array}{l}\text { Motor } \\
\text { difficulties }\end{array}$ & 5.2 & 8500 & 5.4 & $45 \mathrm{del}$ & 11 \\
\hline 6 & Motor delay & 6.3 & 6000 & 7.3 & $54 \mathrm{del}$ & 9.8 \\
\hline 7 & $\begin{array}{l}\text { Motor } \\
\text { difficulties }\end{array}$ & 7.9 & 15424 & 8.6 & $\begin{array}{l}\text { 21-23 } \\
\text { dup }\end{array}$ & 10 \\
\hline 8 & $\begin{array}{l}\text { Motor } \\
\text { difficulties }\end{array}$ & 2.9 & 11470 & 4.0 & 3-7 del & NA \\
\hline 9 & Motor delay & 2.4 & 16000 & 2.4 & 8-12 del & NA \\
\hline 10 & Global delay & 1.8 & 14042 & 1.9 & 45-54 del & NA \\
\hline 11 & Global delay & 2.8 & 30524 & 2.8 & $46-50 \mathrm{del}$ & NA \\
\hline 12 & $\begin{array}{l}\text { Co-ordination } \\
\text { and behavioural } \\
\text { problems }\end{array}$ & 6.0 & 12922 & 6.2 & $45 \mathrm{del}$ & NA \\
\hline 13 & $\begin{array}{l}\text { Motor } \\
\text { difficulties }\end{array}$ & 5.2 & 18269 & 5.8 & c. $580 \mathrm{C}>\mathrm{T}$ & NA \\
\hline
\end{tabular}

Abbreviations: LOA, loss of ambulation; NA, not applicable as still ambulant.

During the 21-year period of the programme, a total of 72 boys with DMD have been identified (56 screen positives, 13 false negatives, 2 whose parents declined screening and 1 case where the card was marked as female) giving an incidence of 1:5136 for DMD in Wales.

\section{DISCUSSION}

Using the bloodspot CK assay protocol described here, we have screened 343170 boys in Wales (United Kingdom), during a 21-year period. To date, this is the most comprehensive and one of the longest 
running newborn bloodspot screening programmes for DMD in the world. The longest running programme based in West Germany, screened 537000 boys during a 34-year period and was also terminated in 2011 because of financial reasons. ${ }^{33}$ The DMD screening test in Germany had to be paid for privately by parents, resulting in a low uptake of the test, with approximately $5 \%$ of all boys born in Germany being screened between 1997 and 2011.

Testing bloodspots collected between days 5 and 8 of life and using a cutoff of $250 \mathrm{U} / \mathrm{l}, 79$ (54\%) of those boys who screened positive for the bloodspot CK test had a normal serum CK when re-tested at 6-8 weeks, giving a FPR of $0.023 \%$. However, if we include those boys with BMD and other dystrophies $(n=89)$, the FPR for this programme was $0.026 \%$. The FPR reported from other programmes ranges from 0.02 to $3.36 \% .{ }^{11}$ The highest rates are due to lower CK cutoffs being used and the screening of bloodspot samples collected from infants $<72 \mathrm{~h}$ of age, where CK activities are significantly increased because of birth trauma. ${ }^{34}$ Numerous causes of false positives cases arose because of birth trauma, congenital hypothyroidism, Pompe disease and other conditions, have been reported. ${ }^{11,15,29}$

The fact that approximately $50 \%$ of the screen positives are transients would suggest that the cutoff of $\geq 250 \mathrm{U} / \mathrm{l}$ should be increased. Using the quantitative bloodspot CK data from 1998 to 2011, applying a cutoff of $\geq 371 \mathrm{U} / \mathrm{l}$ (based on the lowest blood spot $\mathrm{CK}$ result in the DMD group) would have decreased the number of screen-positive cases by 24 (from 93 to 69) during this period. However, identification of 13 false-negative cases during this 21-year period would indicate that raising the threshold is likely to reduce test sensitivity. We are unable to access the bloodspot CK data retrospectively before 2009 and are therefore unable to assess the effect that increasing the cutoff would have on the accuracy of the test.

It has long been recognised that serum CK activities are greatly increased in asymptomatic boys with DMD and, by extension, it was assumed that they would be elevated in the newborn. However, it is important to remember that this increase in blood CK is secondary to the disease process and is not a direct function of the gene defect per se. This may explain why the test sensitivity has been only $\sim 80 \%$ over the 21-year period with 13 false-negative cases. It is very difficult to compare the data from Wales with other programmes as the vast majority of these were small pilot studies. ${ }^{11,21}$ It is known that blood CK activity is proportional to muscle mass and another possible explanation for the false-negative results is that those infants had low birth weights or were small for gestational age. However, in a data set of 3346 boys we found no association between bloodspot CK activity with birth weight and gestational age. The number of false-negative cases observed may simply be due to the insensitivity of the analytical test utilised to screen those samples collected at days 5 to 8 of life.

To reduce diagnostic delay it is vital to identify boys with DMD as early as possible. The mean age at diagnosis of our false-negative group at the time of investigation and the initial serum CK test and at definitive diagnosis was 3.95 and 4.72 years, respectively. These findings are comparable to those observed elsewhere in the United Kingdom. ${ }^{34}$ It is evident that the age at diagnosis over time in the UK population has not changed significantly in the last 20 years. ${ }^{3}$

The reported incidence of 1:4046 for DMD in Wales for the birth cohort 1971-1986, ${ }^{35}$ before the commencement of this screening programme, is higher than the incidence of 1:5136 in the Wales birth cohort 1990-2011. The avoidance of further affected boys in families where the first affected child is diagnosed early is an important consequence of screening, resulting in part from the deferral of future pregnancies when a child is found to have a serious illness and in part from parental decisions to use prenatal diagnostic testing to enable the selective termination of affected pregnancies. ${ }^{27}$ There is some evidence from the Wales study that families do re-assess reproductive patterning as a result of an earlier diagnosis, with 10 out of 12 highrisk families having had prenatal testing in subsequent pregnancies. ${ }^{11}$

The apparently lower incidence of DMD following implementation of the screening programme, however, may have other causes that include chance (ie, sampling effects), the existence of other falsenegative cases still undiagnosed, and the fact that a comprehensive, research-led clinical genetics service was established for DMD families in Wales ahead of many other countries and ahead of many other regions within United Kingdom. It is not possible to attribute the full effect to the newborn screening programme; indeed, the effect of newborn screening on the overall incidence of DMD will be expected to decline as family size continues to decline.

Our laboratory used a bloodspot CK cutoff of $\geq 250 \mathrm{U} / \mathrm{l}$ derived from bloodspots collected at days 5 to 8 of life. Various cutoffs have been utilised (eg, > $300 \mathrm{U} / \mathrm{l}$ in Germany; ${ }^{15}>500 \mathrm{U} / \mathrm{l}$ in Antwerp, Belgium $;^{20}>750 \mathrm{U} / \mathrm{l}$ in Ohio, $\mathrm{USA}^{21}$ ). The difference in cutoffs used is due to several factors; lack of standardisation as different sources of CK have been used, different day of collection of blood spots $\left(<48 \mathrm{~h},^{21}\right.$ to $>4$ weeks of age $\left.\mathrm{e}^{15}\right)$, varying use of assay reaction parameters; including temperatures and type of reaction monitoring (kinetic and end point) have been used to test for bloodspot CK.

It should also be recognised that the bloodspot CK assays that have been used in this programme and in others are nonspecific in that these assays measure total activity. CK is an isoenzyme and several different forms exist (CK-MM, CK-MB and CK-BB). CK-MM is the predominant form present in skeletal muscle and is elevated in patients with muscular dystrophies. ${ }^{36}$ The DMD screening programme in Germany using a total CK activity assay identified numerous false positives ( 1 in 4000) because of a benign autosomal dominant anomaly that results in the overexpression of the CK-BB isoenzyme in erythrocytes. ${ }^{37}$ The development of an immunoassay to detect the CK-MM isoform in blood spots would improve both the sensitivity and specificity and enable robust high-throughput bloodspot screening using standardised reagents and laboratory analysers.

The protocol used here was designed to fit in with the current health-care infrastructure in the United Kingdom, and the re-testing of those boys at 6-8 weeks for serum CK following a positive blood spot CK removes the need for genetic studies and/or muscle biopsy in the false-positive group. The use of the serum CK test at 6-8 weeks of age is a good diagnostic test to discriminate those cases of muscular dystrophy from the transient cases (Table 1). Many pilot studies have taken a similar approach to our protocol. Recently, a pilot study from Ohio, USA ( $n=37649$ ) using blood spots collected before $48 \mathrm{~h}$ of life utilised a two-tier approach of blood spot CK analysis followed by whole-genome amplification and sequencing of all exons on those samples with an increased CK. ${ }^{21}$ This protocol is more readily compatible with the pattern of maternal and child health-care delivery in the United States, with a less developed community midwifery service, and has only just become feasible following the development of a rapid and accurate technique that allows the direct sequence analysis of a large poly-exonic gene such as the DMD gene encoding dystrophin.

It is interesting to compare this approach with that adopted in the newborn screening programmes for cystic fibrosis (CF). In those programmes, the first-tier test is also protein-based (an immunoassay for immunoreactive trypsin) and the second-tier test is molecular. This could be introduced many years earlier than for DMD for several reasons: (i) a small number of mutations accounts for a substantial 
proportion of mutant alleles, (ii) these common mutations can be readily detected by simpler, PCR-based molecular tests and (iii) there is a simple physiological assay (the sweat test) available that is noninvasive and can readily distinguish between those infants who are healthy carriers of CF and those who are affected by the disease. This set of differences means that a very similar, two-tier (protein then DNA) screening programme could be introduced nearly two decades sooner in the case of CF than DMD. The Wales CF protocol has a sensitivity of $94.7 \%$, a PPV of $50 \%$ and a FPR of $0.03 \%$ - not dissimilar to the current DMD protocol outlined here.

To date, only a few studies have reported on the number of false positives and outlined approaches to reduce these by increasing the CK cutoff used, ${ }^{21}$ collecting a second sample for analysis ${ }^{15}$ and including a DNA analysis step. ${ }^{21}$ The advantage of this long-term study is the follow-up and assessment of false-negative cases and this current protocol highlights a potential problem of measuring blood spot CK activity as the first-line test for screening.

If the new treatments are effective in improving outcome in boys with DMD then a strong argument exists for newborn screening. However, before wide scale implementation of blood spot screening can take place, further research is needed to develop a more specific and sensitive assay for blood spot $\mathrm{CK}$ and to further assess the feasibility of a two-tier protocol using CK followed by DNA studies on the newborn blood spot sample collected within the first week of life. Furthermore, to maintain the validity of such screening tests appropriate assay standardisation and the re-introduction of an external quality assurance scheme would be essential.

\section{CONFLICT OF INTEREST}

The authors declare no conflict of interest.

\section{ACKNOWLEDGEMENTS}

We thank Derek Rees, Rhiannon Haynes and Laz Lazarou for Laboratory assistance. We also thank Dr Sharon Hillier and Dr David Elliman for critical reading of the manuscript. It should be acknowledged that the financial support for this programme was initially provided by the Muscular Dystrophy Group of Great Britain and the Wellcome Foundation.

1 Emery AE: Population frequencies of inherited neuromuscular diseases-a world survey. Neuromusc Disord 1991; 1: 19-29.

2 Mohamed K, Appleton R, Nicolaides P: Delayed diagnosis of Duchenne muscular dystrophy. Eur J of Paed Neurol 2000; 4: 219-223.

3 Ciafaloni E, Fox DJ, Pandya S et al: Delayed diagnosis in Duchenne muscular dystrophy: data from the Muscular Dystrophy Surveillance, Tracking, and Research Network (MD STARnet). J Pediatr 2009; 155: 380-385.

4 Bushby K, Hill A, Steele J: Failure of early diagnosis in symptomatic duchenne muscular dystrophy. Lancet 1999; 353: 557.

5 Parsons EP, Clarke AJ, Bradley DM: Developmental progress in Duchenne muscular dystrophy: lessons for earlier detection. Eur J Paed Neurol 2004; 8: 145-153.

6 Firth M, Gardner-Medwin D, Hosking G, Wilkinson E: Interviews with parents of boys suffering from Duchenne muscular dystrophy. Dev Med Child Neurol 1983; 25 : 466-471.

7 Zellweger H, Antonik A: Newborn screening for DMD. Pediatrics 1975; 55: 30-34.

8 Orfanos AP, Naylor EW: A rapid screening tests for Duchenne muscular dystrophy using dried blood specimens. Clin Chim Acta 1984; 138: 264-274.
9 Smith R, Rogers M, Bradley D, Sibert J, Harper P: Screening for Duchenne muscular dystrophy. Arch Dis Child 1989; 64: 1017-1021.

10 Firth MA: Diagnosis of Duchenne muscular dystrophy: experiences of parents of sufferers. Br Med J (Clin Res Ed) 1983; 286: 700-701.

11 Bradley DM, Parsons E: Newborn screening for Duchenne muscular dystrophy. Semin Neonatol 1998; 3: 27-34.

12 Drummond LM, Veale AM: Muscular dystrophy screening. Lancet 1978; 1: 1258-1259.

13 Skinner R, Emery AE, Scheuerbrandt G, Syme J: Feasibility of neonatal screening for DMD. J Med Genet 1982; 19: 1-3.

14 Plauchu H, Cordier MP, Carrier HN et al: Systematic neonatal detection of Duchenne's muscular dystrophy. Results after 10 years' of experience in Lyons (France). J Genet Hum 1987; 35: 217-230.

15 Scheuerbrandt G, Lovgren T, Mortier W: Screening for Duchenne muscular dystrophy —an improved screening test for creatine kinase and its application in an infant screening program. Muscle Nerve 1986; 9: 11-23.

16 Greenberg CR, Rohringer M, Jacobs HK et al: Gene studies in newborn males with DMD detected by neonatal screening. Lancet 1988; 2: 425-428.

17 Naylor EW: New technologies in newborn screening. Yale J Biol Med 1991; 64: 21-24.

18 Hauser E, Toifl K, Mad A, Bittner R: The incidence of Duchenne muscular dystrophy in Eastern Austria. The controversy regarding CK screening. Wien Klin Wochenschr 1993; 105: 433-436.

19 Drousiotou A, loannou P, Georgiou T et al: Neonatal screening for Duchenne muscular dystrophy: a novel semiquantitative application of the bioluminescence test for creatine kinase in a pilot national program in Cyprus. Gene. Test 1998; 2: 55-60.

20 Eyskens F, Philips E: Newborn screening for Duchenne muscular dystrophy. The experience in the province of Antwerp. Neuromuscul Disord 2006; 16: 721-721.

21 Mendell JR, Shilling C, Leslie ND et al: Evidence based path to newborn screening for Duchenne muscular dystrophy. Ann Neurol 2012; 71: 304-313.

22 Ross LF: Screening for conditions that do not meet the Wilson and Jungner criteria: the case of Duchenne muscular dystrophy. Am J Med Genet 2006; 140: 914-922.

23 Moxley 3rd RT, Pandya S: Weekend high-dosage prednisone: a new option for treatment of Duchenne muscular dystrophy. Neurology 2011; 77: 416-417.

24 Manzur AY, Kuntzer T, Pike M, Swan A: Glucocorticoid corticosteroids for Duchenne muscular dystrophy. Cochrane Database Syst Rev 2008; 23: CD003725.

25 Bushby K, Finkel R, Birnkrant DJ et al: DMD Care Considerations Working Group. Diagnosis and management of Duchenne muscular dystrophy, part 1: diagnosis, and pharmacological and psychosocial management. Lancet Neurol 2010; 9: 77-93.

26 Eagle M, Baudouin SV, Chandler C et al: Survival in Duchenne muscular dystrophy: improvements in life expectancy since 1967 and the impact of home nocturnal ventilation. Neuromuscul Disord 2002; 12: 926-929.

27 Bombard Y, Miller FA, Hayeems RZ, Avard D, Knoppers BM: Reconsidering reproductive benefit through newborn screening: a systematic review of guidelines on preconception, prenatal and newborn screening. Eur J Hum Genet 2010; 18: 751-760.

28 Cirak S, Arechavala-Gomeza V, Guglieri M et al: Exon skipping and dystrophin restoration in patients with Duchenne muscular dystrophy after systemic phosphorodiamidate morpholio oligomer treatment: an open-label, phase 2, dose escalation study. Lancet 2011; 378: 595-605.

29 Bradley DM, Parsons EP, Clarke AJ: Experience with screening newborns for Duchenne muscular dystrophy in Wales. Br Med J 1993; 306: 357-360.

30 Parsons E, Bradley DM, Clarke A: Disclosure of Duchenne muscular dystrophy after newborn screening. Arch Dis Child 1996; 174: 550-553.

31 Parsons EP, Clarke AJ, Hood K, Bradley DM: Feasibility of a change in service delivery: the case of optimal newborn screening for Duchenne muscular dystrophy. Community Genet 2000; 31: 17-23.

32 Parsons EP, Clarke AJ, Hood K, Lycett E, Bradley DM: Newborn screening for Duchenne muscular dystrophy: a psychosocial study. Arch Dis Child Fetal Neonatal Ed 2002; 86: F91-F95.

33 Scheuerbrandt G: End of the infant screening program for the early detection of Duchenne muscular dystrophy in Germany. 8th December 2011; http://community. parentprojectmd.org/profiles/blogs/update-from-gunter-scheuerbrandt. Accessed 18 June 2012.

34 Gilboa N, Swanson JR: Serum creatine kinase in normal newborns. Arch Dis Child 1976; 51: 283-285

35 Norman AM, Rogers C, Sibert JR, Harper PS: Duchenne muscular dystrophy in Wales: a 15 year study, 1971 to 1986 . J Med Genet 1989; 26: 560-564.

36 Arenas J, Diaz V, Liras G et al: Activities of creatine kinase and its isoenzymes in serum in various skeletal muscle disorders. Clin Chem 1988; 34: 2460-2462.

37 Arnold H, Wienker TF, Hoffmann MM et al: High levels of brain-type creatine kinase activity in human platelets and leukocytes: a genetic anomaly with autosomal dominant inheritance. Blood Cells Mol Dis 2012; 15: 62-67. 